\title{
When to be discrete: the importance of time formulation in understanding animal movement
}

\author{
Brett T McClintock ${ }^{*}$, Devin S Johnson ${ }^{1}$, Mevin B Hooten², Jay M Ver Hoef ${ }^{3}$ and Juan M Morales ${ }^{4}$
}

\begin{abstract}
Animal movement is essential to our understanding of population dynamics, animal behavior, and the impacts of global change. Coupled with high-resolution biotelemetry data, exciting new inferences about animal movement have been facilitated by various specifications of contemporary models. These approaches differ, but most share common themes. One key distinction is whether the underlying movement process is conceptualized in discrete or continuous time. This is perhaps the greatest source of confusion among practitioners, both in terms of implementation and biological interpretation. In general, animal movement occurs in continuous time but we observe it at fixed discrete-time intervals. Thus, continuous time is conceptually and theoretically appealing, but in practice it is perhaps more intuitive to interpret movement in discrete intervals. With an emphasis on state-space models, we explore the differences and similarities between continuous and discrete versions of mechanistic movement models, establish some common terminology, and indicate under which circumstances one form might be preferred over another. Counter to the overly simplistic view that discrete- and continuous-time conceptualizations are merely different means to the same end, we present novel mathematical results revealing hitherto unappreciated consequences of model formulation on inferences about animal movement. Notably, the speed and direction of movement are intrinsically linked in current continuous-time random walk formulations, and this can have important implications when interpreting animal behavior. We illustrate these concepts in the context of state-space models with multiple movement behavior states using northern fur seal (Callorhinus ursinus) biotelemetry data.
\end{abstract}

Keywords: Animal location data, Diffusion, Movement model, Random walk, State-space model, Switching behavior, Telemetry

\section{Introduction}

Animal movement is at the heart of many important ecological processes and considered essential for a better understanding of population dynamics, animal behavior, and the impacts of global change. However, movement is a complex process modulated by many factors acting at different spatial and temporal scales. Our ability to study animal movement has been bolstered by recent advances in animal-borne biologging technology that have permitted the collection of detailed location and biotelemetry data [1-3]. The quality and quantity of information from these devices is rapidly increasing, and there has been a recent flood in the development of

\footnotetext{
* Correspondence: brett.mcclintock@noaa.gov

${ }^{1}$ National Marine Mammal Laboratory, NOAA-NMFS Alaska Fisheries Science Center, Seattle, WA 98115, USA

Full list of author information is available at the end of the article
}

sophisticated statistical models that use these data for model-based inferences about animal movement and associated behaviors [4-8].

This myriad of new methods for analyzing movement data can make the selection of any particular method (or model) a difficult task, particularly for ecologists and wildlife biologists without formal statistical training. This poses a dilemma because ecologists and biologists constitute the vast majority of scientists collecting the very data for which these methods were developed. The complexities of animal movement and location data require sophisticated analytical techniques, but we believe that the inconsistent mathematical and statistical jargon used to describe these methods may be discouraging their

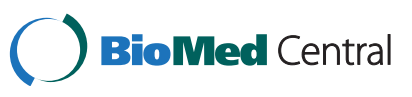

(c) 2014 McClintock et al.; licensee BioMed Central Ltd. This is an Open Access article distributed under the terms of the Creative Commons Attribution License (http://creativecommons.org/licenses/by/4.0), which permits unrestricted use, distribution, and reproduction in any medium, provided the original work is properly credited. The Creative Commons Public Domain Dedication waiver (http://creativecommons.org/publicdomain/zero/1.0/) applies to the data made available in this article, unless otherwise stated. 
widespread application by non-statisticians. In our experience, the greatest source of confusion among practitioners, both in terms of implementation and biological interpretation, seems to be the distinction between continuous- and discrete-time formulations of the movement process.

Here we briefly review several of the model-based (non-phenomenological) approaches for analyzing animal location data that have been proposed in recent years. We then focus on how time is formulated in these movement process models, establish some common terminology (see Table 1), elucidate the differences and similarities among them, and identify some potential advantages and limitations. We also present novel mathematical results (see Does a continuous- or discretetime formulation really matter?) refuting the overly simplistic view that discrete- and continuous-time conceptualizations are merely different means to the same end in terms of inferences about animal movement. We then illustrate these concepts in the context of state-space models with multiple movement behavior states using northern fur seal (Callorhinus ursinus) movement data collected in the Pribilof Islands of Alaska, USA.

\section{Review}

Characterization of the movement process

Regardless of the underlying statistical framework, most analyses of animal location data that are based on hierarchical movement models consist of two components: a mechanistic model for the movement process and a statistical model for the observation process. Although earlier methods ignored error in the location of observations $[5,9,10]$, most contemporary approaches simultaneously model both the movement process and observation process using a so-called' "state-space" framework $[6,8,11,12]$.

Recent technological advances (e.g., GPS) are making location measurement error less of a concern, and this has allowed greater focus on the development of more realistic (and biologically meaningful) models for the movement process. These developments primarily differ

Table 1 Glossary

\begin{tabular}{|c|c|c|}
\hline Term & Definition & Synonyms \\
\hline Behavioral state & $\begin{array}{l}\text { A discrete (and typically latent) behavior associated with a specific } \\
\text { type of movement. }\end{array}$ & Behavior; behavioral mode \\
\hline Brownian motion & $\begin{array}{l}\text { A simple random walk in continuous time, i.e., a diffusion model } \\
\text { with no centralizing tendency. }\end{array}$ & Wiener process \\
\hline Central tendency & $\begin{array}{l}\text { A tendency to move back towards a central location (e.g., the } \\
\text { center of a home range or patch) as a result of directed } \\
\text { movement. }\end{array}$ & Mean-reverting \\
\hline Correlated movement & $\begin{array}{l}\text { Short-term directional persistence resulting from a tendency to } \\
\text { continue moving in a similar direction (or velocity) as previous } \\
\text { moves. }\end{array}$ & \\
\hline Directed movement & $\begin{array}{l}\text { Systematic, non-random movement in a particular direction. } \\
\text { Directed movement associated with a particular location or } \\
\text { gradient, such as a "center of attraction," can result in long-term } \\
\text { directional persistence and/or central tendency. }\end{array}$ & $\begin{array}{l}\text { Biased or oriented movement } \\
\text { (discrete time); drift or advection } \\
\text { (continuous time) }\end{array}$ \\
\hline Directional persistence & A tendency for successive movements to be in a similar direction. & \\
\hline Hidden Markov model & $\begin{array}{l}\text { A special class of state-space models with a finite number of } \\
\text { hidden (e.g., behavioral) states. }\end{array}$ & \\
\hline Markov process & $\begin{array}{l}\text { A stochastic process where state transitions are dependent only } \\
\text { on the current state (first-order Markov process) or current and } \\
\text { immediately previous states (higher-order Markov process). }\end{array}$ & \\
\hline Multistate model & $\begin{array}{l}\text { A mixture of random walk models corresponding to different } \\
\text { movement behavior states. }\end{array}$ & Mixture model, switching model \\
\hline $\begin{array}{l}\text { Ornstein-Uhlenbeck } \\
\text { (OU) process }\end{array}$ & $\begin{array}{l}\text { A diffusion model with centralizing tendency that accounts for } \\
\text { dependence between observations. With no central tendency, } \\
\text { Brownian motion is obtained as a limiting case. }\end{array}$ & \\
\hline Random walk & $\begin{array}{l}\text { Given an initial starting position, a mathematical model for } \\
\text { generating a stochastic movement trajectory in space. Random } \\
\text { walks are often Markov processes and can be formulated in } \\
\text { discrete or continuous time. They have no directional } \\
\text { persistence or bias. }\end{array}$ & \\
\hline State-space model & $\begin{array}{l}\text { A conditionally specified hierarchical model consisting of a latent } \\
\text { system process model and an observation model. }\end{array}$ & \\
\hline
\end{tabular}


in the spatio-temporal conceptualization of the movement process, including discrete-time and discretespace [13-15], discrete-time and continuous-space [5,6], continuous-time and discrete-space [16,17], and continuoustime and continuous-space [8,9] movement process models (see Table 2). Although time formulation in continuous space is our primary focus henceforth, discrete-space movement models are often employed in the absence of detailed location data (e.g., capture-mark-recapture studies e.g., [14,16]), or resource selection studies in heterogeneous environments e.g., [17]. Latent behaviors associated with different types of movement can also be treated as continuous [18] or discrete $[5,6,19,20]$ states among which individuals transition in response to changes in their internal and external environment. Other approaches go a step further by attempting to combine "macroscopic" resource selection models with "microscopic" discrete- or continuous-time movement process models [7,21-27].

Before proceeding, we note that hierarchical discretetime, continuous-space movement process models are often referred to as "state-space" models in the literature. This is not a misnomer. However, based on conventional time series jargon, any approach that simultaneously accounts for the system process (i.e., the movement process) and the observation process through time qualifies as a state-space model. In this sense, all of the hierarchical modeling approaches above employ state-space methods. In the contemporary statistical literature, state-space models are now more commonly referred to as hierarchical models; "hierarchical" because the data arise from a probability distribution that depends on a latent process, which, in turn, is modeled stochastically [34,35]. We also note that discrete-time movement models where each behavioral state is associated with a distinct random walk $[5,6,20,30]$ can be considered as hidden Markov models, a special class of state-space models with a finite number of latent states [36].

In general, animal movement occurs in continuous time but we observe it at fixed discrete-time intervals. Thus, continuous-time models are conceptually and theoretically appealing, but in practice it is perhaps more intuitive to interpret movement in discrete intervals (e.g., turning angle and step length per unit time). It is easier to conceptualize the movement process as a series of steps and turns sampled from particular distributions than to deal with partial differential equations. This may in part explain why the methodological development and application of discrete-time models has thus far exceeded that of continuous-time models.

Whether in discrete or continuous time, most mechanistic movement process models are based on correlated random walks. In discrete time, correlated movement is typically modeled with non-uniform turning angle distributions,

Table 2 Summary of conventional mechanistic movement process models based on spatiotemporal formulation (time and space), movement metric, types of movement that are accounted for (directed or correlated), and accommodation of multiple movement behavior states using multistate models

\begin{tabular}{|c|c|c|c|c|c|c|}
\hline Time & Space & Metric & Directed & Correlated & Multistate & Reference \\
\hline discrete & discrete & position & NA & $\mathrm{NA}$ & yes & [13-15] \\
\hline discrete & continuous & position & yes & no & yes & {$[28]$} \\
\hline discrete & continuous & position & yes & no & no & {$[7]$} \\
\hline discrete & continuous & velocity & no & yes & yes & [6] \\
\hline discrete & continuous & step length & no & yes & yes & [18] \\
\hline discrete & continuous & step length and turning angle & no & yes & yes & {$[5]$} \\
\hline discrete & continuous & step length and bearing & yes & no & no & [29] \\
\hline discrete & continuous & step length and bearing & yes & yes & yes & {$[20,30]$} \\
\hline continuous & discrete & position & yes & no & yes & [16] \\
\hline continuous & discrete & position & yes & yes & no & {$[17]$} \\
\hline continuous & discrete & velocity & yes & yes & yes & [19] \\
\hline continuous & continuous & position & yes & no & no & [10] \\
\hline continuous & continuous & position & yes & no & yes & {$[4,9,31]$} \\
\hline continuous & continuous & $\begin{array}{l}\text { step length and turning angle } \\
\text { velocity }\end{array}$ & no & yes & yes & [32] \\
\hline continuous & continuous & velocity & no & yes & no & [33] \\
\hline continuous & continuous & velocity & yes & yes & no & [8] \\
\hline continuous & continuous & velocity & yes & yes & yes & [31] \\
\hline
\end{tabular}

Example references are also provided. 
usually with mean of zero, which result in short-term directional persistence between successive time steps. The more highly correlated movement exhibits turning angles tending towards zero [5,6]. In continuous time, correlated movement can be expressed through a special type of diffusion model that accounts for dependence between locations, the Ornstein-Uhlenbeck (OU) process $[4,10]$. The OU process is essentially a continuoustime random walk with a tendency to drift towards a central location. Using an OU process to model movement velocity instead of locations, Johnson et al. [8] developed a correlated random walk model that is a continuous-time analog to the discrete-time model of Jonsen et al. [6].

Both discrete- and continuous-time random walk models can incorporate directed (or oriented) movement, but this is often referred to as "biased" movement in discrete-time models [20,37] and "drift" or "advection" in continuoustime models $[4,10]$. Directed movements are typically associated with specific locations in space, such as "centers of attraction" or "centers of repulsion," and can be used to model a general tendency towards the center of a home range $[7,10]$ or patch $[4,20,31]$. Thus, directional persistence can result from directed movements, but the longterm directional persistence that can result from directed movement is different from the short-term directional persistence associated with a correlated random walk [38]. Under directed movement, longer-term directional persistence results from an individual being constantly pulled towards (or pushed away from) a particular location or gradient (without explicit consideration of the direction of previous movements).

Without correlated movements, the discrete-time models of Morales et al. [5] and Jonsen et al. [6] reduce to simple random walks. Without directed movements, the discretetime model of McClintock et al. [20] reduces to the correlated random walk model of Morales et al. [5]. The OU process models of Dunn and Gipson [10], Blackwell [4,9], Johnson et al. [8], and Harris and Blackwell [31] reduce to Brownian motion (i.e., a continuous-time simple random walk), using a mathematical limit argument. We note that because the directional persistence in a correlated random walk decays exponentially as the time lapse increases, correlated random walks can be approximated at larger scales with a simple diffusion model [16].

To incorporate both correlated and directed movement, the expected direction of movement must reflect a trade-off between short-term directional persistence and the strength of bias towards (or away from) a center of attraction (or repulsion). This has been examined in discrete time by modeling the expected direction as a weighted average of the strength of bias in the direction of the center of attraction and the previous movement direction [20,37]. Although a similar approach has yet to be thoroughly investigated in continuous time, this would be akin to modeling the drift parameter of an OU process as a function of both directed and correlated movements.

\section{The metrics of movement}

Movement metrics also differ among the aforementioned approaches by specifying the movement process on the positions themselves $[7,9,28]$ or on derived quantities, such as the differences between consecutive locations (i.e., velocities) $[6,8,19,32,33]$, step lengths [18], step lengths and turning angles [5], or step lengths and bearings $[20,29]$ (see Table 2). These movement metrics are important for model specification and interpretation. For example, by modeling velocity, the discrete-time model of Jonsen et al. [6] and the continuous-time model of Johnson et al. [8] induce dependence between the speed and direction of movement, so that long steps are possible when turning angles are small, resulting in higher-order auto-correlations than found in standard correlated random walks [5,20]. Although Blackwell $[4,9]$ models position and Johnson et al. [8] model velocity, the speed and direction of movement are intrinsically linked through the drift process of these continuous-time models (see Does a continuous- or discrete-time formulation really matter?). By modeling turning angles independent of step lengths in discretetime, Morales et al. [5] could investigate correlated (but not directed) movements independent of speed. By modeling bearings using a similar discrete-time movement process model, McClintock et al. [20] could simultaneously investigate both correlated and directed movements independent of speed.

\section{Does a continuous- or discrete-time formulation really matter?}

Outside of fitting them to data and empirically assessing differences, it is not immediately apparent how alternative time formulations of movement models differ analytically. In fact, continuous- and discrete-time formulations are often over simplistically viewed as merely different means to the same end. But this is not the case, and we derive a partial translation here to compare continuous- and discrete-time formulations with a common and intuitive language: step length and bearing.

Kobayashi et al. [39] provides the following necessary result for two independent normally-distributed random variables, $A$ and $B$. If

$$
[A, B]=\mathcal{N}\left(\mu_{A}, \sigma^{2}\right) \mathcal{N}\left(\mu_{B}, \sigma^{2}\right),
$$

then the distance from the origin, $L=\sqrt{A^{2}+B^{2}}$, has a Rice distribution, $R\left(\mu, \sigma^{2}\right)$, where $\mu=\sqrt{\mu_{A}^{2}+\mu_{B}^{2}}=\|\boldsymbol{\mu}\|$ is the distance from the origin to the center of the bivariate normal distribution, $\sigma^{2}$ is a variance parameter, 
and $\mathcal{N}()$ is the Normal probability density function. The Rice distribution is a generalization of the Rayleigh distribution (for $\mu \neq 0$ ) whose expected value increases with increasing values of $\mu$. Further, the bearing $\theta=\tan ^{-1}$ $(B / A)$, has the conditional von Mises distribution

$$
[\theta \mid L=l]=V M(\omega, \kappa)=\frac{\mathrm{e}^{\kappa \cos (\theta-\omega)}}{2 \pi I_{0}(\kappa)},
$$

where $\kappa=l \mu / \sigma^{2}, \omega=\tan ^{-1}\left(\mu_{B} / \mu_{A}\right)$, and $I_{0}()$ is the modified Bessel function of the first kind and of order 0 . The von Mises distribution is symmetric and centered on the angle $\omega$, and dispersion decreases with increasing $\kappa$ values.

We can now translate a time step of the continuoustime correlated random walk (CTCRW) model of Johnson et al. [8] to a discrete-time step length and bearing. First, the transformation of the bivariate velocity process to speed (distance unit per time unit) and direction is given by

$$
l_{t}=\left\|v_{t}\right\|
$$

and

$$
\theta_{t}=\tan ^{-1}\left(V_{y, t} / V_{x, t}\right)
$$

The resulting distributions are obtained by applying the results in Kobayashi et al. [39] to the CTCRW velocity model equations (see Eqs. 3 and 4 in Continuous-time formulation below). Using the velocity process transformation, the location step length $S_{t}=\sqrt{\left(X_{t+1}-X_{t}\right)^{2}+\left(Y_{t+1}-Y_{t}\right)^{2}}$ and bearing $\phi_{t}=\tan ^{-1}\left\{\left(Y_{t+1}-Y_{t}\right) /\left(X_{t+1}-X_{t}\right)\right\}$ are distributed as

$$
\begin{aligned}
& {\left[S_{t} \mid l_{t}, Z_{t}=z\right]=R\left(l_{t}\left(1-e^{-\beta_{z}}\right) / \beta_{z}, q_{z, t}\right)} \\
& {\left[\phi_{t} \mid S_{t}, l_{t}, Z_{t}=z\right]=\operatorname{VM}\left(\theta_{t}, S_{t} l_{t} e^{-\beta_{z}} / q_{z, t}\right),}
\end{aligned}
$$

where $Z_{t}$ is the latent behavioral state, and $q_{z, t}$ is the $(1,1)$ (or, $(2,2)$ as they are the same) entry of the covariance matrix for the velocity process $\left(\mathbf{Q}_{z, t}\right)$.

There are now notable differences that one can easily distinguish between the continuous and discrete formulations for step length and bearing distributions. First, unlike the discrete-time model (see Eqs. 1 and 2 in Discrete-time formulation below), the step length and bearing of the continuous-time model are clearly correlated. As step length increases the distribution of the bearing becomes more concentrated around $\theta_{t}$, the latent velocity bearing. Second, given a constant state process, step lengths are independent in the discretetime formulation. However, in the CTCRW model step lengths are still correlated via the auto-correlated speed process, $l_{t}$. Thus, unlike the discrete-time model, the CTCRW maintains not just directional persistence, but persistence in speed as well. Note that this result does not depend on latent behavioral state $\left(Z_{t}\right)$ and holds for movement models with a single behavioral state.

We emphasize that these results are not simply attributable to the fact that the CTCRW model is based on an integrated OU velocity. They hold analogously for continuous-time models which use OU process models for position directly [4,9,31], even if $X_{t}$ and $Y_{t}$ are modeled independently (i.e., by setting the offdiagonal elements of the covariance matrix for the bivariate $\mathrm{OU}$ process to zero). Using the same result from Kobayashi et al. [39], the distributions of the step length and bearing of an OU process directly modeling position, with central location $\boldsymbol{\mu}=\left(\mu_{x}, \mu_{y}\right)$, are

$$
\begin{aligned}
& {\left[S_{t} \mid Z_{t}=z\right]=R\left(D_{t}(\boldsymbol{\mu})\left\{e^{-\beta_{z}}-1\right\}, \sigma_{t}^{2}\right)} \\
& {\left[\phi_{t} \mid S_{t}, Z_{t}=z\right]=\operatorname{VM}\left(\theta_{t}, S_{t}\left\{e^{-\beta_{z}}-1\right\}\right)}
\end{aligned}
$$

where $D_{t}(\boldsymbol{\mu})$ and $\theta_{t}$ are respectively the distance and bearing from the current position to the central location, and $\sigma_{t}^{2}$ is the variance of the OU process at time $t$. One can see that the OU model directly applied to the positions still maintains correlation between step length and bearing. Moreover, it also possesses the (potentially undesirable) quality that movement rate depends on distance from the point of attraction, thus necessitating rapid movement that slows as the animal approaches the central location.

\section{Potential advantages and disadvantages}

Given the various ways by which similar movement properties can be expressed using either discrete- or continuous-time process models, some potential advantages and disadvantages are evident. Although animal movement clearly occurs in continuous time, discretetime models are often viewed as more intuitive, and perhaps the biological interpretation of instantaneous movement parameters in continuous time (e.g., those related to OU processes and other diffusion models) can in practice be discouraging to applied ecologists wishing to use or extend continuous-time methods.

Notably, discrete-time models that simultaneously incorporate multiple latent movement behavior states, Markov state-switching, correlated movements, and directed movements have already been developed and fitted to data $[5,6,20]$. For example, Morales et al. [5] used a discrete-time random walk mixture model to examine time allocations and transition probabilities between two latent movement behaviors in elk: a longstep, directionally-persistent "exploratory" state and a short-step, negatively-correlated (i.e., with animals tending to move in the opposite direction of the previous move) "encamped" state. Similarly, Jonsen et al. [6] investigated analogous "transit" and "foraging" movement behavior 
states in seals. Also using seal data, McClintock et al. [20] developed a biased, correlated random walk mixture model with five latent movement behavior states allowing for directed and exploratory movement among foraging and haul-out locations.

Similar applications of multistate mixture models have yet to appear in continuous-time (but see Example: northern fur seal). Blackwell [9] assumed movement behavior states were known, and Johnson et al. [8] assumed states were defined by known covariates, hence neither of these approaches included an estimation framework for both latent movement states and switching behavior. Hanks et al. [19] extended the framework of Johnson et al. [8] and Hooten et al. [17] to accommodate inhomogeneous movement characteristics along the movement path using a change-point model. However, because this approach does not explicitly incorporate distinct movement behavior states or state-switching mechanisms with direct biological interpretation, post hoc cluster analyses were used to identify potential movement behavior states. Harris and Blackwell [31] recently described a continuous-time multistate mixture modeling framework, but fitting these models is challenging, and they have yet to be demonstrated using real data. Part of the difficulty of multistate mixture models in continuous time is due to the underlying relationships these models typically impose on the movement characteristics (e.g., speed or directional persistence) commonly used to distinguish movement behavior states (see Does a continuous- or discrete-time formulation really matter? and Example: northern fur seal). Because multistate models are of great practical importance for investigating time allocations to different behaviors (i.e., "activity budgets"), this currently remains an advantage of discrete-time models.

Two important disadvantages of discrete-time models are related to the necessary discretization of the movement path into a finite number of temporally-regular time steps [40]. The time step length must be specified $a$ priori, but inferences about animal movement from a discrete-time analysis are not time scale-invariant. For example, inferences about bumblebee movement characteristics from discrete-time analyses using 30-second versus 30 -minute time steps would likely be dramatically different. The 30-second analysis would reveal fine-grain movement properties but could potentially mask coarsergrain properties. The 30 -minute analysis could reveal coarse-grain properties, but would completely miss finegrain properties. The specification of time step length in a discrete-time analysis is therefore critical and requires very careful consideration [41-43], and it is particularly important that the time step is chosen to match the scale at which behavioral decisions are made [40]. A major advantage of continuous-time models is that they avoid dependence on a particular timescale. Within reasonable limits, a continuous-time analysis will yield the same results regardless of the temporal resolution of observations; if so desired, movement properties from a continuoustime analysis may be summarized a posteriori for time steps of any length. However, we note that for any continuous- or discrete-time approach to be useful, the temporal resolution of the observed data must be relevant to the specific movement behaviors of interest.

Discrete-time movement models can also be more computationally demanding than continuous-time models. Unless observations exactly match the regular time steps required of a discrete-time model, the movement path must be predicted at temporally-regular intervals. Perfectly observed, temporally-regular observations are very rare in animal telemetry data (especially for marine species). For longer time series, this can result in thousands of additional location parameters that must be estimated. As movement process models incorporate more details and realism, model fitting becomes more complex. This is particularly true for multistate mixture models. Therefore, once multistate model development and fitting in continuous time has caught up with that in discrete time, the computational advantages of continuous-time formulations are likely to be significant.

\section{Example: northern fur seal}

To illustrate the concepts elaborated above in the context of state-space models with latent movement behavior states, we apply comparable multistate movement models in discrete and continuous time to a northern fur seal track in the Pribilof Islands of Alaska, USA. The animal was a nursing female equipped with a Mk10-AF satellite tag from Wildlife Computers (see [44] for full study deployment details). The Mk10-AF tag has both Fastloc GPS and time-depth recording capabilities. Using both location and diving activity data, we wish to identify and characterize three latent movement behavior states: "resting," "foraging," and "transit". We define foraging (state F) as movement that is characteristic of area restricted searches and includes foraging dives, where a foraging dive must have a max depth $>5 \mathrm{~m}$ and at least 5 changes in vertical direction (i.e., sinuosities or "wiggles"). The sinuosities are a characteristic of the animal chasing prey during the dive. We define transit (state $\mathrm{T}$ ) as predominantly travelling with little to no foraging dives, noting that seals may opportunistically feed while travelling. Resting (state R) is defined by types of movement that do not fall under foraging or transit states, including resting at haulouts and resting at sea. In terms of trajectory, we would expect speeds to be low during resting and low to moderate during foraging, with little directional persistence. During transit, we would expect higher speeds and greater directional persistence. 
The diving activity data were summarized as the number of foraging dives for each of $N=242$ 1-hour time steps between 7-17 October 2007. Although diving data were logged continuously, location data were obtained opportunistically at 15 -minute intervals. There are therefore frequent missing location data due to an inability to obtain locations while the seal was underwater. Because the tag possessed GPS capabilities, rather than ARGOS technology, we expect location measurement error to be minimal. The raw location data consist of 241 observations during a single foraging trip (Figure 1), with $40 \%$ of the 1-hour time steps containing no observed locations.

\section{Discrete-time formulation}

With the location data being temporally irregular, a discrete-time analysis requires that the movement path be estimated at regular time steps. We chose 1-hour time steps to exactly match the temporal resolution of the foraging dive data. Using the same state-space formulation as McClintock et al. [20], for time step $t=1, \ldots, N$, and observation $i=1, \ldots, k_{t}$, we relate the irregularly observed locations $\left(x_{t, i}, y_{t, i}\right)$ to the temporally regular model locations $\left(X_{t}, Y_{t}\right)$ using

$$
x_{t, i}=\left(1-j_{t, i}\right) X_{t-1}+j_{t, i} X_{t}+\epsilon_{x_{t, i}}
$$

and

$$
y_{t, i}=\left(1-j_{t, i}\right) Y_{t-1}+j_{t, i} Y_{t}+\epsilon_{y_{t, i}}
$$

where $j_{t, i} \in[0,1)$ is the proportion of the time interval between locations $\left(X_{t-1}, Y_{t-1}\right)$ and $\left(X_{t}, Y_{t}\right)$ at which the $i^{\text {th }}$ observation between times $t$ - 1and $t$ was obtained, $\left[\epsilon_{x_{t, i}}\right]=\mathcal{N}\left(0, \sigma_{x}^{2}\right), \quad\left[\epsilon_{y_{t, i}}\right]=\mathcal{N}\left(0, \sigma_{y}^{2}\right), \quad[\ldots]$ indicates the probability density function for the random variable in brackets, and $\mathcal{N}()$ is the Normal (Gaussian) density. Time steps with no observations (i.e., $k_{t}=0$ ) do not contribute to the observation model.

We then model movement between the temporally regular locations using a multistate correlated random walk model $[5,20]$. Specifically, we assume that, conditional on the behavioral state, $Z_{t}$, the step length at time $t, S_{t}$, is distributed as

$$
\begin{aligned}
{\left[S_{t} \mid \boldsymbol{a}, \boldsymbol{b}, Z_{t}=z\right]=} & \operatorname{Weibull}\left(a_{z}, b_{z}\right)=\frac{b_{z}}{a_{z}}\left(\frac{S_{t}}{a_{z}}\right)^{b_{z}-1} \\
& \times \exp \left[-\left(\frac{S_{t}}{a_{z}}\right)^{b_{z}}\right]
\end{aligned}
$$

where $S_{t} \geq 0, z \in\{R, F, T\}$ is the unknown latent behavioral state, and $a_{z}$ and $b_{z}$ are state-dependent scale and shape parameters, respectively. The Weibull distribution is popular for modeling step length because of its flexibility; it has fat tails when $b_{z}<1$, reduces to an exponential distribution when $b_{z}=1$, has exponential tails when $b_{z}>1$, and can resemble a normal distribution when $b_{z} \approx$ 3.4. The bearing of movement, $\phi_{t}$, is modeled with the wrapped Cauchy distribution

$$
\begin{aligned}
& {\left[\phi_{t} \mid \phi_{t-1}, \boldsymbol{\rho}, Z_{t}=z\right]=w \operatorname{Cauchy}\left(\phi_{t-1}, \rho_{z}\right)} \\
& =\frac{1-\rho_{z}^{2}}{2 \pi\left[1+\rho_{z}^{2}-2 \rho_{z} \cos \left(\phi_{t}-\phi_{t-1}\right)\right]}
\end{aligned}
$$

where $0 \leq \phi_{t}<2 \pi, \phi_{t-1}$ is the previous bearing, and $-1<\rho_{z}<1$ is the state-dependent dispersion parameter. Unfamiliar to most non-statisticians, the wrapped Cauchy distribution converges to a uniform distribution over the circle as $\rho_{z}$ goes to zero. As $\rho_{z}$ goes to 1 (or -1 ),

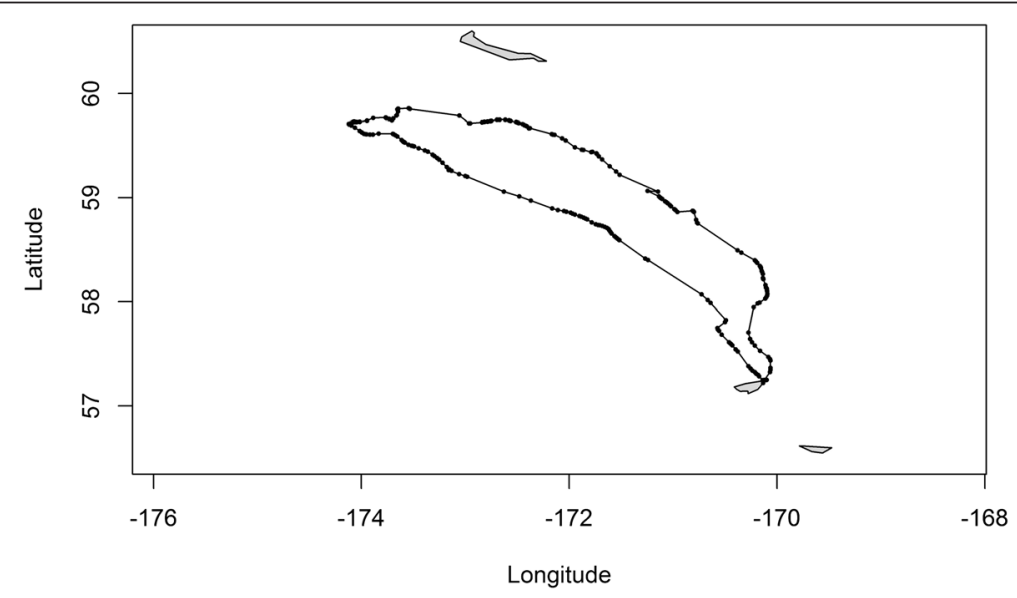

Figure 1 Observed locations during a foraging trip 10-17 October 2007 for a northern fur seal that hauls out in the Pribilof Islands, Alaska. 
the distribution tends to a point mass concentrated towards (or away from) the previous bearing. Standard correlated movement is typically modeled with the wrapped Cauchy distribution by constraining $0 \leq \rho_{z}<1[5,45]$.

It can be difficult to distinguish resting, foraging, and transit states for seals based on trajectory alone [45], particularly because northern fur seals can forage opportunistically while travelling and will often rest at sea or in the vicinity of breeding rookeries. We therefore incorporate the number of foraging dives during each time step, $\delta_{t}$, to help inform the foraging state. Specifically, we assume

$$
\left[\delta_{t} \mid \lambda, Z_{t}=z\right]=\operatorname{Poisson}\left(\lambda_{z}\right)
$$

with the constraints $\lambda_{R}=0$ and $\lambda_{F}>\lambda_{T}$. This model therefore assumes a priori that time steps with foraging dives are never assigned to resting, and steps with relatively many foraging dives are more likely to be assigned to foraging than transit. Note that by constraining $\lambda_{F}>\lambda_{T}$, we still allow some possibility for steps with foraging dives to be assigned to transit.

Finally, we model switches between behavior states as a first-order Markov process. We assign the conditional distribution to the latent state variable $Z_{t}$

$$
\left[Z_{t} \mid \boldsymbol{\psi}, Z_{t-1}=z\right]=\text { Categorical }\left(\psi_{z, R}, \psi_{z, F}, \psi_{z, T}\right)
$$

where for $z, z^{\prime} \in\{R, F, T\}, \psi_{z, z^{\prime}}$ is the probability of switching from state $z$ at time $t-1$ to state $z^{\prime}$ at time $t$.

Using Bayesian analysis methods, the joint posterior distribution for our state-space model in discrete time is

$$
\begin{aligned}
& {\left[\boldsymbol{a}, \boldsymbol{b}, \boldsymbol{\rho}, \boldsymbol{\lambda}, \boldsymbol{\psi}, \sigma_{x}^{2}, \sigma_{y}^{2}, X_{0}, Y_{0}, \boldsymbol{\phi}, \mathbf{S}, \mathbf{Z} \mid \mathbf{x}, \mathbf{y}, \boldsymbol{\delta}\right]} \\
& \left.\propto \prod_{t=1}^{N}\left\{\left[S_{t} \mid \boldsymbol{a}, \boldsymbol{b}, Z_{t}\right)\right]\left[\phi_{t} \mid \phi_{t-1}, \boldsymbol{\rho}, Z_{t}\right)\right]\left[\delta_{t} \mid \boldsymbol{\lambda}, Z_{t}\right]\left[Z_{t} \mid \boldsymbol{\psi}, Z_{t-1}\right] \\
& \left.\times \prod_{i=1}^{k_{t}}\left[x_{t, i}, y_{t, i} \mid \sigma_{x}^{2}, \sigma_{y}^{2}, X_{0}, Y_{0}, \boldsymbol{\phi}_{[1: t]}, \mathbf{S}_{[1: t]}\right]\right\} \\
& \times[\boldsymbol{a}][\boldsymbol{b}][\boldsymbol{\rho}][\boldsymbol{\psi}][\boldsymbol{\lambda}]\left[\sigma_{x}^{2}\right]\left[\sigma_{y}^{2}\right]\left[X_{0}, Y_{0}\right]
\end{aligned}
$$

where $\left(X_{0}, Y_{0}\right)$ is the initial (latent) location. Note that, conditional on $Z_{t}$, this discrete-time model assumes step length, bearing, and the number of foraging dives are independent. Weakly informative priors were used for all parameters, including the conjugate priors $\left[\sigma_{x}^{2}\right]=\Gamma^{-1}$ $(0.01,0.01), \quad\left[\sigma_{y}^{2}\right]=\Gamma^{-1}(0.01,0.01), \quad\left[\lambda_{z}\right]=\Gamma(0.01,0.01)$ for $z \in\{F, T\}$, and $\left[\boldsymbol{\psi}_{z}\right]=\operatorname{Dirichlet}(1,1,1)$ for $z \in\{R, F, T\}$, where $\Gamma()$ and $\Gamma^{-1}()$ are the gamma and inverse gamma probability density functions, respectively. For $\left[X_{0}, Y_{0}\right]$, we specified a joint uniform prior over the region defined by the Bering Sea. We specified a maximum sustainable speed of $3 \mathrm{~m} / \mathrm{s}$, such that $S_{t} \leq 10800 \mathrm{~m}$, with
$\left[a_{z}\right]=\operatorname{Unif}(0,10800),\left[b_{z}\right]=\operatorname{Unif}(0,30)$, and $\left[\rho_{z}\right]=$ Unif $(0,1)$ for $z \in\{R, F, T\}$. Similar to McClintock et al. [20,45], we used a Metropolis-within-Gibbs Markov chain Monte Carlo algorithm written in the $\mathrm{C}$ programming language [46] to obtain samples from the posterior distribution, performing pre- and post-processing in $\mathrm{R}$ via the .C interface [47]. The only notable difference from the MCMC algorithm for the individual-level model of McClintock et al. [45] results from our model for $\delta_{t}$, for which the conjugate prior on $\lambda_{z}$ yields the full conditional distributions $\left[\lambda_{F} \mid \cdot\right]=\Gamma_{\left(\lambda_{T}, \infty\right)}\left(0.01+\sum_{t=1}^{N} \delta_{t} I_{\left\{Z_{t}=F\right\}}, 0.01+\sum_{t=1}^{N} I_{\left\{Z_{t}=F\right\}}\right)$ and $\left[\lambda_{T} \mid \cdot\right]=\Gamma_{\left(0, \lambda_{F}\right)}\left(0.01+\sum_{t=1}^{N} \delta_{t} I_{\left\{Z_{t}=T\right\}}, 0.01+\sum_{t=1}^{N} I_{\left\{Z_{t}=T\right\}}\right)$, where $\Gamma_{(l, u)}$ is the renormalized gamma density truncated at $l$ and $u, 0 \leq l<u$, and $I()$ is the indicator function. When full conditional distributions were analytically intractable, random walk Metropolis-Hastings parameter updates were used. After initial pilot tuning and burn-in, a single chain of 5 million iterations was attained for posterior summaries. The algorithm required approximately 3 hours to run on a machine running 64-bit Windows $7(3.4 \mathrm{GHz}$ Intel Core i7 processor, 16Gb RAM).

Estimated activity budgets to the three movement behavior states were 0.28 (95\% HPDI: 0.22-0.37) to resting, $0.36(0.26-0.39)$ to foraging, and $0.36(0.29-0.45)$ to transit (Figure 2a). Estimated state transition probabilities were $\hat{\psi}_{R, R}=0.81(0.71-0.92), \hat{\psi}_{F, F} \cdot=0.78(0.67-0.88)$, and $\hat{\psi}_{T, T}=0.78$ (0.65-0.89), with state-switches more likely to occur between foraging $\hat{\psi}_{F, T}=0.15$ (0.05-0.27) and transit $\hat{\psi}_{T, F}=0.14(0.06-0.22)$. The bivariate posterior densities for step length and turning angle (Figure 3a) indicate some opportunistic foraging during travelling, with foraging movements often exhibiting high speed and directional persistence typically associated with transit. As expected, time steps with $>1$ foraging dives were rarely assigned to the transit state (Figure 4a). Also as expected, we found lower speeds and less directional persistence during resting movements and higher speed and more directional persistence during transitory movements.

The estimated error (in meters) for the observation process model was similar between longitude $\left(\sigma_{x}=472\right.$; $360-596)$ and latitude $\left(\sigma_{y}=489 ; 381-617\right)$ coordinates. Although relatively small, these errors are larger than would typically be expected of GPS location measurement error. We therefore suspect the additional error is attributable to deviations from the simple linear model used to relate the temporally irregular observed locations to temporally-regular predicted locations.

\section{Continuous-time formulation}

We analyzed the same fur seal data set using a continuoustime model to assess what inferential differences might result by extending the correlated random walk (CRW) 

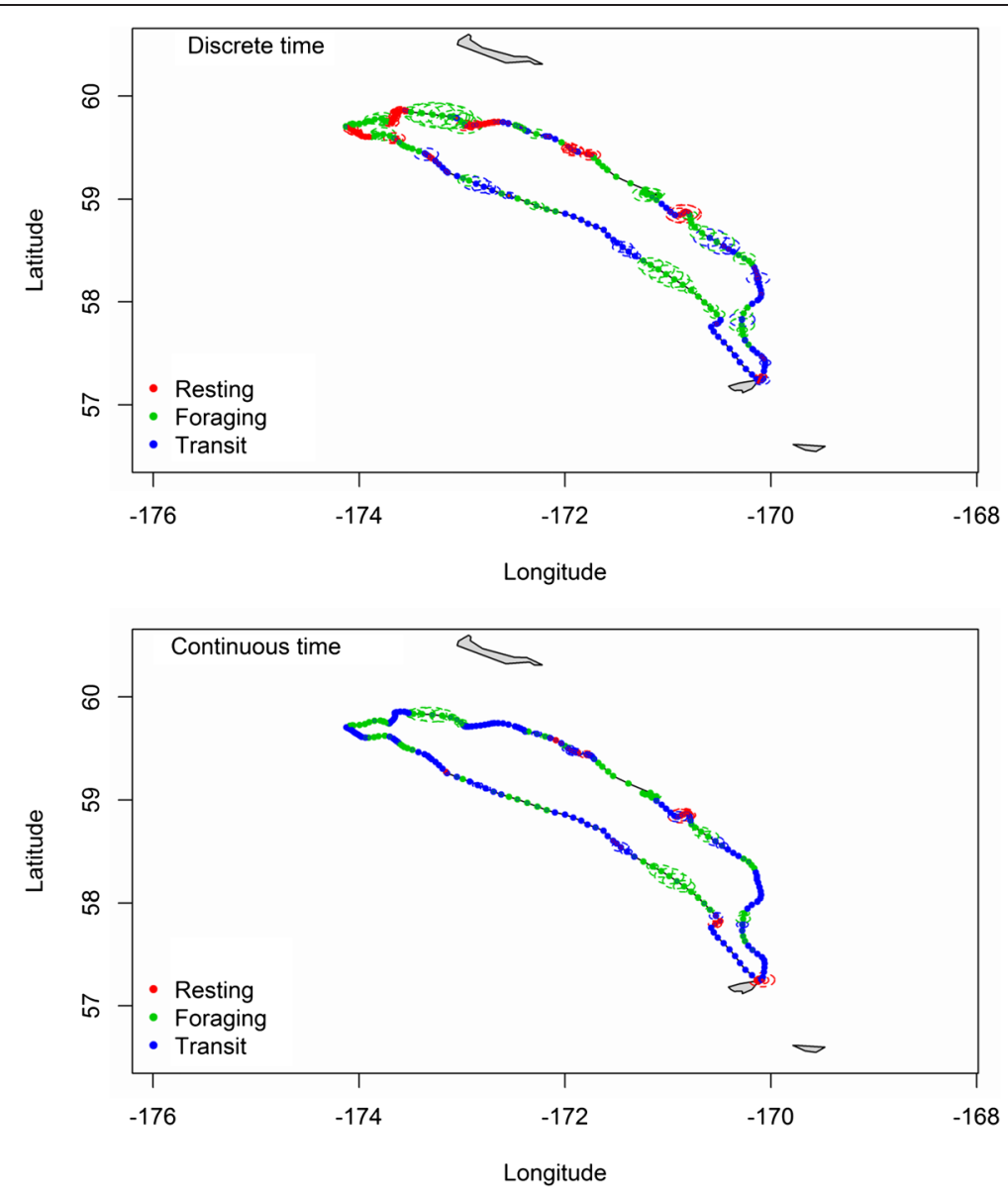

Figure 2 Estimated path and movement behavior states during a foraging trip of a northern fur seal that hauls out in the Pribilof Islands, Alaska. Results are presented for discrete- and continuous-time movement process models. Estimated movement states for the predicted locations correspond to "resting" (red), "foraging" (green), and "transit" (blue) movement behavior states. Uncertainty in the state assignments (<95\% posterior probability) are indicated by hollow circles within predicted locations. Uncertainty in predicted locations are indicated by $95 \%$ credible bands (dashed lines).

models of Jonsen et al. [6] (discrete-time, latent states) and Johnson et al. [8] (continuous-time, state model with known covariates) to a continuous-time CRW model with latent states. The continuous-time correlated random walk (CTCRW) is described by modeling the velocity (instantaneous rate of change) of movement with a bivariate Ornstein-Uhlenbeck (OU) process. The OU process is the continuous-time version of the bivariate autoregressive model Jonsen et al. [6] use to model position difference. The CTCRW locations are then modeled by integrating the velocity process (i.e., the positions are the solution to the stochastic differential equation used to model velocity).

To make the inference comparable between each analysis, we maintained the same hourly structure for the transitions of behavior states. Thus, the models $\left[Z_{t} \mid \psi Z_{t-1}=z\right]$ and $\left[\delta_{t} \mid \lambda Z_{t}=z\right]$ are the same as in the previous discretetime analysis with the minor technical change that the state $Z_{t}$ is assumed to be held constant within the interval $[t, t+$
1). Also, we use the notation $t_{i}$ to represent the time of the $i$ th observed location in the interval $[t, t+1)$.

The CTCRW model is defined by a stochastic differential equation model of velocity $\boldsymbol{v}_{t_{i}}=\left(V_{x, t_{i}}, V_{y, t_{i}}\right)$ at time $t_{i}$, such that

$$
V_{c, t_{i+1}}=\gamma_{c}+e^{-\beta_{t} \Delta_{\mathrm{i}}}\left(V_{c, t_{i}}-\gamma_{c}\right)+\zeta\left(\Delta_{\mathrm{i}}\right)
$$

for each coordinate axis $c \in\{x, y\}$, where $t \leq t_{i}<t_{i+1} \leq t+1$, $\Delta_{\mathrm{i}}=t_{i+1}-t_{i}, \gamma_{c}$ is the mean velocity (or drift), $\beta_{t}$ is an autocorrelation parameter, $\zeta\left(\Delta_{\mathrm{i}}\right)=\mathcal{N}\left(0, \sigma_{t}^{2}\left[1-\exp \left(-2 \beta_{t} \Delta_{\mathrm{i}}\right)\right] / 2 \beta_{t}\right)$, and $\sigma_{t}$ is a parameter controlling the overall variability in velocity. The solution to this autoregressive differential equation is the location $\boldsymbol{\mu}_{t_{i}}=\left(X_{t_{i}}, Y_{t_{i}}\right)$. Johnson et al. [8] provide details to illustrate that the CTCRW model can be formulated as a linear, Gaussian state-space model that allows efficient calculation of the CTCRW likelihood. For $t \leq$ $t_{i}<t_{i+1} \leq t+1$, observation $\mathbf{y}_{t_{i}}=\left(x_{t_{i}}, y_{t_{i}}\right)$, and the vector 

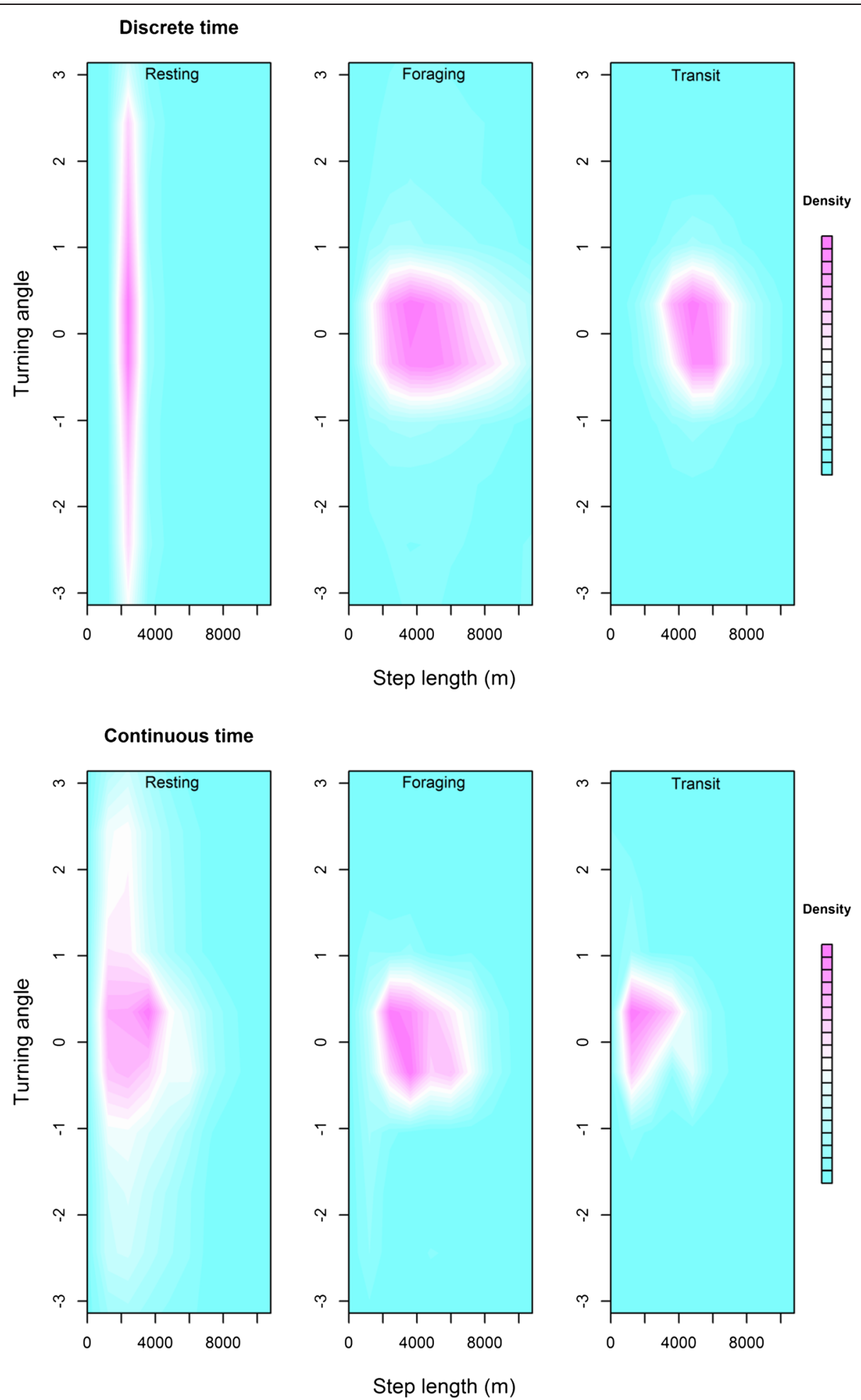

Figure 3 Estimated bivariate densities of northern fur seal step lengths and turning angles for three distinct movement behavior states ("resting", "foraging", and "transit") based on discrete- and continuous-time movement process models with 1-hour time steps. For both models, step lengths and turning angles were calculated from the estimated paths shown in Figure 2. 


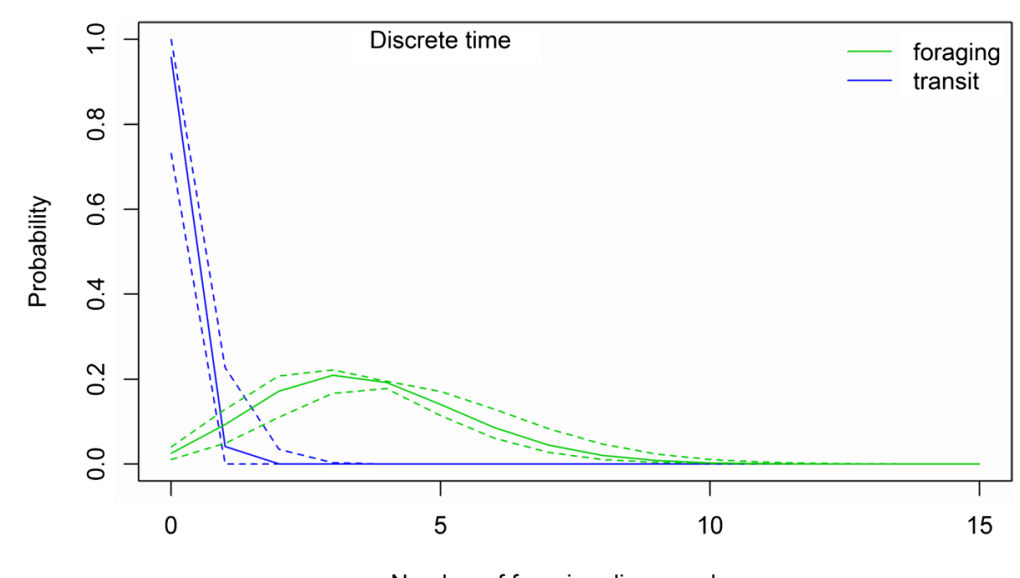

Number of foraging dives per hour

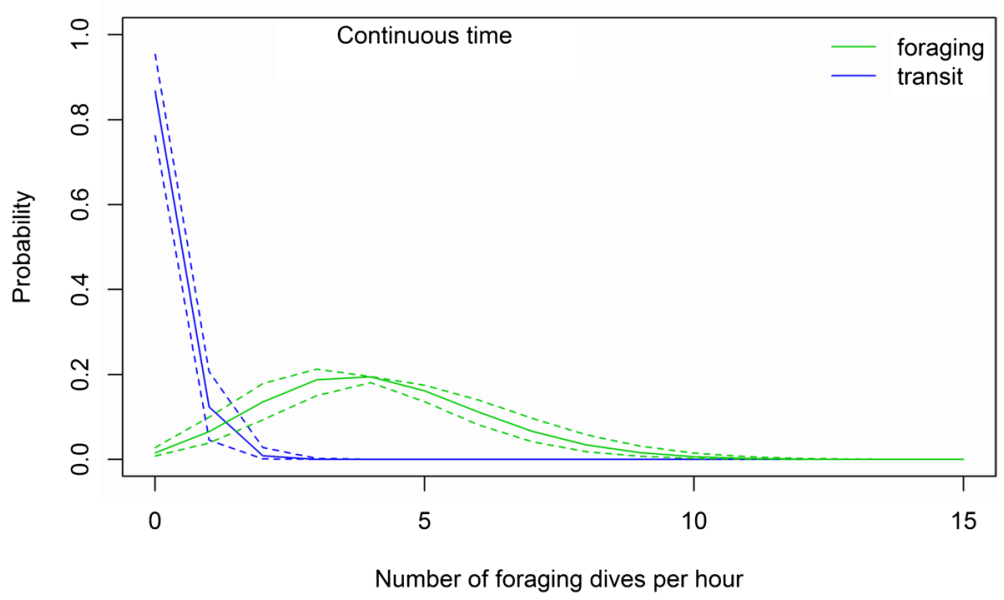

Figure 4 Hourly probabilities for the number of foraging dives by a northern fur seal while in the foraging and transit states based on discrete- and continuous-time movement process models. Foraging dives were defined as dives with a max depth $>5 \mathrm{~m}$ with at least 5 sinuosities (i.e., "wiggles"). Probabilities were calculated from the estimated Poisson distribution for $\delta_{t}$ based on posterior samples for $\lambda_{F}$ and $\lambda_{T}$. Dashed lines indicate 95\% highest posterior density intervals.

of the true location and velocity process $\boldsymbol{\alpha}_{t_{i}}=\left(\boldsymbol{\mu}_{t_{i}}, \boldsymbol{v}_{t_{i}}\right)$, the state-space model is given by

$$
\begin{gathered}
\mathbf{y}_{t_{i}}=\boldsymbol{\mu}_{t_{i}}+\boldsymbol{\varepsilon}_{t_{i}} \\
\boldsymbol{\alpha}_{t_{i+1}}=\mathbf{T}_{z, t_{i}} \boldsymbol{\alpha}_{t_{i}}+\boldsymbol{\eta}_{z, t_{i}}
\end{gathered}
$$

where $\left[\boldsymbol{\varepsilon}_{t_{i}}\right]=\mathcal{N}\left(\mathbf{0}, \tau^{2} \mathbf{I}\right)$ and $\left[\boldsymbol{\eta}_{z, t_{i}}\right]=\mathcal{N}\left(\mathbf{0}, \mathbf{Q}_{z, t_{i}}\right)$. The entries of $\mathbf{T}_{z, t_{i}}$ and $\mathbf{Q}_{z, t_{i}}$ are functions of $\Delta_{\mathrm{i}}$ and the movement parameters $\beta_{t}$ and $\sigma_{t}$ (see [8] for details), and as in the discrete-time analysis, the movement parameters depend on the latent state $Z_{t}=z$ via $\beta_{t}=\beta_{z}$ and $\sigma_{t}=\sigma_{z}$.

We used an MCMC sampler for Bayesian inference of movement parameters and states. Similar to Johnson et al. [8], we assumed no drift (i.e., $\gamma_{c}=0$ ) and similar movement processes in both coordinates (i.e., $\beta_{c, t}=\beta_{t}$ and $\sigma_{c, t}=\sigma_{t}$ for $\left.c \in\{x, y\}\right)$. The same priors were used for all common variables between the two analyses (e.g., diving rates, behavior states). For the CTCRW movement parameters, we used vague priors on the log scale with the following constraints: $\beta_{R}>\beta_{F}>\beta_{T}$ and $\sigma_{R}<\sigma_{F}<\sigma_{T}$. These constraints imply that movement is typically faster and more correlated as one moves from $R$ to $T$. The flat prior $[\log \tau]>10 \mathrm{~m}$ was used for the measurement error parameter. The sampler was custom coded in $\mathrm{R}$ [47] making use of the FORTRAN coded CTCRW likelihood and posterior track simulation in the $\mathrm{R}$ package crawl [48]. The CTCRW likelihood computed via the Kalman filter allowed us to sample from the marginal posterior distribution of the states and movement parameters without having to sample the unobserved $\boldsymbol{\alpha}_{t}$ values. The sampled posterior distribution is given by

$$
\begin{aligned}
& {[\boldsymbol{\beta}, \boldsymbol{\sigma}, \tau, \boldsymbol{\lambda}, \boldsymbol{\psi}, \mathbf{Z} \mid \mathbf{x}, \mathbf{y}, \boldsymbol{\delta}] \propto[\boldsymbol{\beta}][\boldsymbol{\sigma}][\tau] \prod_{t=1}^{N}\left[\delta_{t} \mid \boldsymbol{\lambda}, Z_{t}\right]\left[Z_{t} \mid \boldsymbol{\psi}, Z_{t-1}\right]} \\
& \times \prod_{t=1}^{N} \prod_{i=1}^{k_{t}}\left[x_{t_{i}}, y_{t_{i}} \mid Z_{t}, \beta_{t}, \sigma_{t}, \tau\right]
\end{aligned}
$$


where the right hand-side of the product is the CTCRW likelihood. Note that the true locations $\left(X_{t_{i}}, Y_{t_{i}}\right)$ and velocities $\left(V_{x, t_{i}}, V_{y, t_{i}}\right)$. have been integrated from the posterior. The benefit of this is that the MCMC sampler for the states and parameters converges more quickly to the approximate posterior distribution. The full algorithm took 66 hours to run (due to coding in $\mathrm{R}$ rather than $\mathrm{C}$ ), however, only 20,000 iterations were necessary to obtain an effective sample of $\geq 4,000$ posterior draws. To compare step lengths and turning angles of the CTCRW model to the discrete time model, we needed a sample of hourly locations. To obtain a posterior sample of $\boldsymbol{\alpha}_{t}, t=1, \ldots, N$, on the hour, the sampling method of Johnson et al. [49] was used at each MCMC iteration as if $\boldsymbol{\alpha}_{t}$ was a derived parameter. From the sampled $\boldsymbol{\alpha}_{t}$ values, step length and turning angle were calculated for comparison to the equivalent discrete-time quantities.

Estimated activity budgets to the three movement behavior states were 0.10 (95\% HPDI: 0.03-0.15) to resting, $0.29(0.23-0.34)$ to foraging, and $0.61(0.53-0.67)$ to transit (Figure 2b). Estimated state transition probabilities were $\hat{\psi}_{R, R}=0.52(0.10-0.86), \hat{\psi}_{F, F}=0.75(0.62$ $0.86)$, and $\hat{\psi}_{T, T}=0.82(0.75-0.89)$. State-switches to transit were most likely, with $\hat{\psi}_{R, T}=0.40(0.09-0.81)$ and $\hat{\psi}_{F, T}=$ 0.23 (0.12-0.35). These are noticeably different from the discrete-time analysis, with much less time spent "resting." The bivariate posterior densities for step length and turning angle (Figure 3b) also reflect this reduction in state $R$, with more small steps associated with the travel state. However, there were also more large steps associated with the resting state. This calls into question the designation of these states as actually "resting" when using the continuous-time multistate movement model. As in the discrete-time analysis, time steps with $>1$ foraging dives were rarely assigned to the transit state (Figure $4 \mathrm{~b}$ ). The estimated error (in meters) for the observation process model was $\hat{\tau}=64 \mathrm{~m}(55 \mathrm{~m}-75 \mathrm{~m})$. Because the observed data linear interpolation does not need to be accounted for, the measurement error variance is noticeably smaller here than in the discrete-time analysis.

Although inferences about time spent foraging were similar between the two approaches, we found considerable differences between the discrete-time and continuoustime formulations with respect to resting and travelling activity. This is counter to the simplistic view that time formulations are merely different means to the same end. The reasons for these differences lie in the underlying relationships of the metrics of movement (speed and directional persistence) that are used to define resting and travelling. Because these metrics are dependent and speed is auto-correlated in the continuous-time model (see Does a continuous- or discrete-time formulation really matter?), the lack of auxiliary information (such as metabolic rate) to help distinguish these movement behavior states induces a tendency for the "resting" state to be associated with sudden switches (or change-points) in movement properties during periods with no foraging dives. In other words, instead of identifying periods of slow movement with no foraging dives as intended, the "resting" state serves to break the momentum of the continuoustime movement process.

Although continuous-time formulations necessarily induce dependence between step length and bearing, the differences between our discrete- and continuous-time analyses are not entirely attributable to time formulation per se. In order to account for short-term directional persistence in continuous time, Johnson et al. [8] used correlation in the velocity process (Jonsen et al. [6] use the same correlation model in discrete time). Whether in continuous or discrete time, the modelling of velocity clearly induces additional dependence between speed and bearing. Correlated random walk models with two latent movement behavior states can be relatively easy to fit in continuous time (D. Johnson, unpublished data) or when modeling velocity in discrete time $[6,50]$. However, the modelling of velocity can make it more difficult to characterize and identify $>2$ distinct movement behavior states with straightforward biological interpretation. While this can be easily avoided in discrete time by modelling step length and bearing independently (as was done here), most continuous-time CRW models are formulated on the velocity process $[8,31]$ (but see [22]).

\section{Conclusions}

Modern tracking and biologging devices allow us to record detailed information on animal location and physiology, thus opening the possibility to better understand the role of movement in population dynamics, animal behavior, and the environment [51,52]. To make the most of these hard-earned data and learn about important aspects of animal movement such as activity budgets, space use, and behavioral responses to landscape features, sophisticated data analysis tools have been proposed. State-space models, where one explicitly accounts for the fact that the observed data arise from a mechanistic or "biological" model that is in turn sampled by an observation model, are currently regarded as the most correct and elegant methods to fit movement models to data [12,52]. We have shown that there exist underappreciated differences among the current available formulations, and although our northern fur seal example focused on state-space models with multiple movement behavior states, our findings have important implications for singlestate mechanistic movement process models, including (discrete-time) step-selection or (continuous-time) partial differential equation resource selection models (e.g., see recent reviews by $[26,27])$. 
Although movement is a continuous-time process, it is perhaps more intuitive to think about (and formulate models for) movement in discrete time. In our experience, practitioners find a discrete-time model (Eqs. 1 and 2) and its parameters easier to interpret than its continuous-time counterpart (Eqs. 3 and 4). As we have demonstrated, current discrete-time formulations also provide both flexibility and feasibility for identifying latent behavioral states and incorporating auxiliary biotelemetry or environmental data to inform these states. However, these advantages of discrete-time models do indeed come at a cost. Because inferences from discretetime models are not time scale-invariant, it is absolutely critical that the chosen time scale between movement steps appropriately matches the animal's behavioral scales and the frequency of observations.

In addition to loss of resolution, when observations are irregular and/or the frequency of observations greatly exceeds that of the chosen time scale, discrete-time models can suffer from additional lack of fit due to the need to discretize the movement path into temporally-regular locations. This was apparent in the magnitudes of the measurement error terms in our northern fur seal example, where the discrete-time model had larger errors than would normally be expected for GPS data. The need for temporally-regular positions for the entire movement path can also make it more difficult to deal with missing data in a discrete-time framework. While this is less of a problem for terrestrial animals, missing data is a major issue for marine animals due to our inability to obtain locations while underwater.

Continuous time is clearly a more natural representation of movement than discrete time. These models are not dependent on any particular time scale and do not require temporally-regular observations. It is therefore far easier to deal with missing data or changing observational frequencies in continuous time. However, as demonstrated by our northern fur seal example and Does a continuous- or discrete-time formulation really matter?, current continuous-time formulations may not be well suited for identifying $>2$ latent movement behavior states. This is unfortunate because the identification of different behaviors, activity budgets, and how these potentially relate to habitat use and demographic parameters is among the most interesting aspects of movement ecology [51].

Although discrete-time approaches thus far have seen greater development and application, we believe further development of continuous-time models is needed to facilitate more widespread application of these models to real data. For example, the continuous formulations of Blackwell [9], Johnson et al. [8], and Harris and Blackwell [31] could potentially be extended to accommodate "stops" where animals can reorient and change movement state, thereby curbing the momentum inherent to these continuous-time movement process models. By overcoming the hurdles identified here and making latent state-switching models more feasible in continuous time, the best of both worlds may soon be within grasp.

\section{Abbreviations}

CRW: Correlated random walk; CTCRW: Continuous-time correlated random walk; MCMC: Markov chain Monte Carlo; OU: Ornstein-Uhlenbeck.

\section{Competing interests}

The authors declare that they have no competing interests.

\section{Authors' contributions}

All authors conceived, drafted, read, and approved the manuscript. BTM conducted the discrete-time analysis of the northern fur seal data. DSJ conducted the continuous-time analysis of the northern fur seal data. All authors read and approved the final manuscript.

\section{Acknowledgements}

The findings and conclusions in the paper are those of the author(s) and do not necessarily represent the views of the National Marine Fisheries Service, NOAA. Any use of trade, firm, or product names is for descriptive purposes only and does not imply endorsement by the U.S. Government. JMM was funded by CONICET and PICT 20110790.

\section{Author details}

${ }^{1}$ National Marine Mammal Laboratory, NOAA-NMFS Alaska Fisheries Science Center, Seattle, WA 98115, USA. ²U.S. Geological Survey, Colorado Cooperative Fish and Wildlife Research Unit, Department of Fish, Wildlife, and Conservation Biology, Department of Statistics, Colorado State University, Fort Collins, CO 80523, USA. ${ }^{3}$ National Marine Mammal Laboratory, NOAA-NMFS Alaska Fisheries Science Center, Fairbanks, AK 99775, USA.

${ }^{4}$ Ecotono, INIBIOMA-CONICET, Universidad Nacional del Comahue, Quintral 1250, Bariloche 8400, Argentina.

Received: 8 July 2014 Accepted: 29 September 2014 Published online: 15 October 2014

\section{References}

1. Cooke SJ, Hinch SG, Wikelski M, Andrews RD, Kuchel L, Wolcott TG, Butler PJ: Biotelemetry: a mechanistic approach to ecology. Trends Ecol Evol 2004, 19:334-343.

2. Wilson RP, Shepard E, Liebsch N: Prying into the intimate details of animal lives: use of a daily diary on animals. Endangered Species Research 2008, 4:123-137.

3. Rutz C, Hays GC: New frontiers in biologging science. Biol Lett 2009, 5:289-292.

4. Blackwell P: Random diffusion models for animal movement. Ecol Model 1997, 100:87-102.

5. Morales JM, Haydon DT, Frair J, Holsinger KE, Fryxell JM: Extracting more out of relocation data: building movement models as mixtures of random walks. Ecology 2004, 85:2436-2445.

6. Jonsen ID, Flemming JM, Myers RA: Robust state-space modeling of animal movement data. Ecology 2005, 86:2874-2880.

7. Christ A, Ver Hoef J, Zimmerman DL: An animal movement model incorporating home range and habitat selection. Environ Ecol Stat 2008, 15:27-38.

8. Johnson DS, London JM, Lea M-A, Durban JW: Continuous-time correlated random walk model for animal telemetry data. Ecology 2008, 89:1208-1215.

9. Blackwell P: Bayesian inference for Markov processes with diffusion and discrete components. Biometrika 2003, 90:613-627.

10. Dunn JE, Gipson PS: Analysis of radio telemetry data in studies of home range. Biometrics 1977, 33:85-101.

11. Anderson-Sprecher R, Ledolter J: State-space analysis of wildlife telemetry data. J Am Stat Assoc 1991, 86:596-602.

12. Patterson TA, Thomas L, Wilcox C, Ovaskainen O, Matthiopoulos J: State-space models of individual animal movement. Trends Ecol Evol 2008, 23:87-94. 
13. Brownie C, Hines JE, Nichols JD, Pollock KH, Hestbeck J: Capture-recapture studies for multiple strata including non-Markovian transitions. Biometrics 1993, 1173-1187.

14. Schwarz CJ, Schweigert JF, Arnason AN: Estimating migration rates using tag-recovery data. Biometrics 1993, 49:177-193.

15. Dupuis JA: Bayesian estimation of movement and survival probabilities from capture-recapture data. Biometrika 1995, 82:761-772.

16. Ovaskainen $\mathrm{O}$, Rekola H, Meyke E, Arjas E: Bayesian methods for analyzing movements in heterogeneous landscapes from mark-recapture data. Ecology 2008, 89:542-554.

17. Hooten MB, Johnson DS, Hanks EM, Lowry JH: Agent-based inference for animal movement and selection. J Agric Biol Environ Stat 2010, 15:523-538.

18. Forester JD, Ives AR, Turner MG, Anderson DP, Fortin D, Beyer HL, Smith DW, Boyce MS: State-space models link elk movement patterns to landscape characteristics in Yellowstone National Park. Ecological Monographs 2007, 77:285-299.

19. Hanks EM, Hooten MB, Johnson DS, Sterling JT: Velocity-based movement modeling for individual and population level inference. PLoS One 2011, 6:e22795.

20. McClintock BT, King R, Thomas L, Matthiopoulos J, McConnell BJ, Morales JM: A general discrete-time modeling framework for animal movement using multistate random walks. Ecological Monographs 2012, 82:335-349.

21. Rhodes JR, McAlpine CA, Lunney D, Possingham HP: A spatially explicit habitat selection model incorporating home range behavior. Ecology 2005, 86:1199-1205.

22. Moorcroft PR, Lewis MA, Crabtree RL: Mechanistic home range models capture spatial patterns and dynamics of coyote territories in Yellowstone. Proc Biol Sci 2006, 273:1651-1659.

23. Moorcroft PR, Barnett A: Mechanistic home range models and resource selection analysis: a reconciliation and unification. Ecology 2008, 89:1112-1119.

24. Forester JD, Im HK, Rathouz PJ: Accounting for animal movement in estimation of resource selection functions: sampling and data analysis. Ecology 2009, 90:3554-3565.

25. Potts JR, Bastille Rousseau G, Murray DL, Schaefer JA, Lewis MA: Predicting local and non-local effects of resources on animal space use using a mechanistic step selection model. Methods Ecol Evol 2014, 5:253-262.

26. Potts JR, Lewis MA: How do animal territories form and change? Lessons from 20 years of mechanistic modelling. Proc Biol Sci 2014, 281:20140231.

27. Thurfjell H, Ciuti S, Boyce MS: Applications of step-selection functions in ecology and conservation. Movement Ecology 2014, 2:4.

28. Jonsen ID, Myers RA, James MC: Robust hierarchical state-space models reveal diel variation in travel rates of migrating leatherback turtles. J Anim Ecol 2006, 75:1046-1057.

29. Tracey JA, Zhu J, Crooks KR: Modeling and inference of animal movement using artificial neural networks. Environ Ecol Stat 2011, 18:393-410.

30. Langrock R, King R, Matthiopoulos J, Thomas L, Fortin D, Morales JM: Flexible and practical modeling of animal telemetry data: hidden Markov models and extensions. Ecology 2012, 93:2336-2342.

31. Harris KJ, Blackwell PG: Flexible continuous-time modelling for heterogeneous animal movement. Ecol Model 2013, 255:29-37.

32. Gurarie $E$, Andrews RD, Laidre KL: A novel method for identifying behavioural changes in animal movement data. Ecol Lett 2009, 12:395-408.

33. Gurarie E, Ovaskainen O: Characteristic spatial and temporal scales unify models of animal movement. Am Nat 2011, 178:113-123.

34. Berliner LM: Hierarchical Bayesian time series models. In Maximum entropy and Bayesian methods. Netherlands: Springer; 1996:15-22.

35. Cressie N, Calder CA, Clark JS, Hoef JMV, Wikle CK: Accounting for uncertainty in ecological analysis: the strengths and limitations of hierarchical statistical modeling. Ecol Appl 2009, 19:553-570.

36. Zucchini W, MacDonald IL: Hidden Markov models for time series: an introduction using R. New York: CRC Press; 2009.

37. Barton KA, Phillips BL, Morales JM, Travis JM: The evolution of an 'intelligent' dispersal strategy: biased, correlated random walks in patchy landscapes. Oikos 2009, 118:309-319.

38. Benhamou S: Of scales and stationarity in animal movements. Ecol Lett 2014, 17:261-272

39. Kobayashi H, Mark BL, Turin W: Probability, Random Processes, and Statistical Analysis. Cambridge University Press Textbooks; 2011.

40. Turchin P: Quantitative analysis of movement: measuring and modeling population redistribution in animals and plants. Massachusetts, USA: Sinauer Associates Sunderland; 1998.

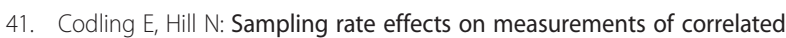
and biased random walks. J Theor Biol 2005, 233:573-588.

42. Breed GA, Costa DP, Goebel ME, Robinson PW: Electronic tracking tag programming is critical to data collection for behavioral time-series analysis. Ecosphere 2011, 2:art10.

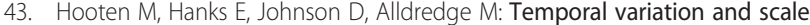
in movement-based resource selection functions. Statistical Methodology 2014, 17:82-98.

44. Kuhn CE, Johnson DS, Ream RR, Gelatt TS: Advances in the tracking of marine species: using GPS locations to evaluate satellite track data and a continuous-time movement model. Mar Ecol Prog Ser 2009, 393:97-109.

45. McClintock BT, Russell DJ, Matthiopoulos J, King R: Combining individual animal movement and ancillary biotelemetry data to investigate population-level activity budgets. Ecology 2013, 94:838-849.

46. Kernighan BW, Ritchie DM, Ejeklint P: The C programming language. Englewood Cliffs: Prentice-Hall; 1988.

47. R Core Team: R: A Language and Environment for Statistical Computing R Foundation for Statistical Computing; 2013.

48. Johnson DS: Crawl: Fit continuous-time random walk models to animal movement data. 2013, R package version 14-1 http://cran.r-project.org/ web/packages/crawl/crawl.pdf.

49. Johnson DS, London JM, Kuhn CE: Bayesian inference for animal space use and other movement metrics. J Agric Biol Environ Stat 2011, 16:357-370.

50. Breed GA, Don Bowen W, Leonard ML: Behavioral signature of intraspecific competition and density dependence in colony-breeding marine predators. Ecology and evolution 2013, 3:3838-3854.

51. Morales JM, Moorcroft PR, Matthiopoulos J, Frair JL, Kie JG, Powell RA, Merril $\mathrm{EH}$, Haydon DT: Building the bridge between animal movement and population dynamics. Philos Trans R Soc Lond B Biol Sci 2010, 365:2289-2301.

52. Schick RS, Loarie SR, Colchero F, Best BD, Boustany A, Conde DA, Halpin PN, Joppa LN, McClellan CM, Clark JS: Understanding movement data and movement processes: current and emerging directions. Ecol Lett 2008, 11:1338-1350.

doi:10.1186/s40462-014-0021-6

Cite this article as: McClintock et al.: When to be discrete: the importance of time formulation in understanding animal movement. Movement Ecology 2014 2:21.

\section{Submit your next manuscript to BioMed Central and take full advantage of:}

- Convenient online submission

- Thorough peer review

- No space constraints or color figure charges

- Immediate publication on acceptance

- Inclusion in PubMed, CAS, Scopus and Google Scholar

- Research which is freely available for redistribution

Submit your manuscript at www.biomedcentral.com/submit
C Biomed Central 